\title{
Matching of the low-x evolution kernels *
}

\author{
V.S. Fadin $^{a \dagger}$, R. Fiore ${ }^{b \ddagger}$, A.V. Grabovsky ${ }^{a \dagger \dagger}$ \\ ${ }^{a}$ Budker Institute of Nuclear Physics, 630090 Novosibirsk, Russia \\ Novosibirsk State University, 630090 Novosibirsk, Russia \\ ${ }^{b}$ Dipartimento di Fisica, Università della Calabria, \\ Istituto Nazionale di Fisica Nucleare, Gruppo collegato di Cosenza, \\ Arcavacata di Rende, I-87036 Cosenza, Italy
}

\begin{abstract}
We demonstrate that the ambiguity of the low-x evolution kernels in the next-to-leading order (NLO) permits one to match the Möbius form of BFKL kernel and the kernel of the colour dipole model and to construct the Möbius invariant NLO BFKL kernel in $N=4$ supersymmetric Yang-Mills theory.
\end{abstract}

${ }^{*}$ Work supported in part by the RFBR grant 0\%-02-00953, in part by the RFBR-MSTI grant 06-02-72041, in part by the INTAS grant 05-1000008-8328 and in part by Ministero Italiano dell'Istruzione, dell'Università e della Ricerca.

${ }^{\dagger} e$-mail address: FADIN@INP.NSK.SU

${ }^{\ddagger}$ e-mail address: FIORE@CS.INFN.IT

${ }^{\dagger \dagger} e$-mail address: A.V.GRABOVSKY@INP.NSK.SU 


\section{Introduction}

This paper is devoted to the investigation of conformal properties of the Balitsky-Fadin-KuraevLipatov (BFKL) equation [1] and the relationship between the BFKL approach and the colour dipole model [2].

The BFKL approach gives the most common basis for the theoretical description of semihard processes in QCD and has a wide field of applications covering scattering processes with arbitrary momentum and colour exchanges. Now the next-to-leading (NLO) corrections to the kernel of the BFKL equation are known both for the forward scattering [3], [4] and for any momentum and colour transfer [5]-[7].

The BFKL approach is based on the gluon reggeization. Originally it was formulated in the momentum representation, and the BFKL kernel was calculated in the space of transverse momenta $\overrightarrow{q_{1}}, \overrightarrow{q_{2}}$ of two interacting reggeized gluons. Later it was recognized that for the case of scattering of colourless objects the BFKL equation possesses remarkable properties, which become mostly apparent in the space of conjugate coordinates $\vec{r}_{1}, \vec{r}_{2}$. It was shown [8] that in this case the BFKL equation can be written in the special representation (in the space of states $|\Psi\rangle$ with the "dipole" property $\langle\vec{r}, \vec{r} \mid \Psi\rangle=0$ ), where the equation is invariant under the conformal (Möbius) transformations of the transverse coordinates. Following Ref. [9] we will call this representation Möbius representation. For brevity, we will also call the BFKL kernel in this representation Möbius kernel, and its form in the coordinate space Möbius (or dipole) form. The Möbius form of the LO BFKL kernel is explicitly conformal invariant [10]. Moreover, it coincides with the kernel of the evolution equation in the colour dipole model.

The colour dipole model is formulated in the coordinate space. Unlike the BFKL approach, it is applicable only to scattering of colorless particles. Its attractive feature is a clear physical interpretation. The model is applied not only at low parton density, but also in the high density regime, where parton fusion is essential [11, and evolution equations become nonlinear. In general, in this regime there is an infinite hierarchy of coupled equations [12]-[14]. In the simplest case, when the target is a large nucleus, it is reduced to the Balitsky-Kovchegov (BK) equation [13]. It was shown [9] that the BK equation appears as a special case of the nonlinear evolution equation which sums the fan diagrams for the BFKL Green's functions in the Möbius representation. Therefore in the LO there is a full agreement between the BFKL approach and colour dipole model.

Recently the NLO corrections to the BK kernel have been calculated [15]-[17] and investigation of inter-relation of the BFKL approach and the colour dipole model in the NLO became possible. A clear understanding of this inter-relation is important for the further development of theoretical description of small-x processes. Not less significant is the understanding of conformal properties of the NLO BFKL kernel in the Möbius representation because the conformal invariance is extremely important for integrability of the BFKL equation. Evidently, in QCD 
the conformal invariance is violated by the running coupling, i.e. by the terms proportional to $\beta$-function. But one could expect that the Möbius form is quasi-conformal, i.e. the conformal invariance is violated only by such terms, and that it remains unbroken in $N=4$ SUSY YangMills One could expect also coincidence of the Möbius form of the BFKL kernel and the kernel of the colour dipole model.

However, the situation is not so simple, because the NLO kernels are not unambiguously defined. Their ambiguity is analogous to the ambiguity of the NLO anomalous dimensions. It is caused by the possibility to redistribute radiative corrections between the kernels and the impact factors.

We prove that this ambiguity permits one to match the Möbius form of the BFKL kernel and the kernel of the colour dipole model and to construct the Möbius invariant NLO BFKL kernel in $N=4$ SUSY.

The NLO corrections consist of quark and gluon parts. The quark part of the BK kernel was found Refs. [13, [16]; the corresponding part of the Möbius form of the BFKL kernel was calculated in Refs. [10, 18]. Taking into account of the ambiguity mentioned above, it was shown there that up to the difference in the renormalization scales the quark parts agree with each other. Moreover, the "abelian" piece of the quark part is conformal invariant [18]. This is especially interesting for the QED Pomeron [19, 20] because this piece is proportional to the total QED kernel.

The Möbius form for the gluon part was obtained in Ref. [21. As well as the quark part, it turned out strikingly simple compared with the gluon part of the BFKL kernel in transverse momentum space. However, it was found that the conformal invariance of this form is broken not only by the terms proportional to $\beta$-function. In principle, this result did not mean that the conformal invariance was broken in $N=4$ SUSY Yang-Mills theory because the field structure of this theory differs from QCD. The extension of the BFKL framework to the supersymmetric theories was started in Ref. 22, where the forward kernel was found for the SUSY N=4 in the space of the Born eigenfunctions and in the momentum space with the dimension $D=4+2 \epsilon$. This analysis has been expanded Ref. the [23, where the Möbius form of the nonforward BFKL kernel was obtained for the supersymmetric theories with arbitrary N. It turned out that this form violates conformal invariance at any N. However, because of the ambiguity discussed above a possibility of existence of a conformal invariant kernel at $N=4$ was not excluded. Also Regge limits of 4-point correlators were studied in $\mathrm{N}=4$ SUSY directly in the coordinate space via conformal invariance and the connection of the analysis to the AdS/CFT duality was discussed in Ref. [24].

The gluon part of the BK kernel calculated in Ref. [17] agreed neither with the Möbius form of Ref. [21], nor with the eigenvalues of the forward BFKL kernel [3, 22]. It was found afterwards that Ref. [17] contained an error, which was corrected in Ref. [25]. The discrepancy of the results of Refs. [17] and [3, 22, 21] was analyzed in detail in Ref. [26]. A special attention 
was paid to the case of the forward scattering. It was shown for this case that with account of the ambiguity of the NLO kernels, of the correction performed in Ref. 25] and of the difference in the renormalization scales, the discrepancy disappeared. Besides this, the functional identity of the forward BFKL kernel in the momentum and Möbius representations in the leading order (LO) was exhibited and its NLO validity in $N=4$ supersymmetric Yang-Mills theory was proved.

Here we demonstrate that with account of the correction of Ref. [25] and the difference in the renormalization scales (it was discussed in detail in Ref. 26] and we will not mention it further) the ambiguity of the NLO kernels permits us to match the gluon parts for the non-forward case also. Moreover, this ambiguity allows us to present the kernel in the form where the conformal invariance is violated only by renormalization. It is especially interesting for the $\mathrm{N}=4$ SUSY Yang-Mills.

Our paper is organized as follows. In the next section, our notation is introduced, the ambiguity of the NLO kernels is briefly discussed and a short overview of the discrepancy between the kernels is given. Section 3 presents the transformation of the BFKL kernel (allowed by the NLO ambiguity) which removes this discrepancy. In section 4 the transformation to the quasi-conformal shape is presented. Section 5 gives the quasi-conformal (conformal at $\mathrm{N}=4$ ) kernels in QCD and SUSY Yang-Mills theories. Section 6 presents our conclusions.

\section{General overview}

Our notation is the same as in Refs. [10, 21]. Thus we denote Reggeon transverse momenta (conjugate coordinates) in initial and final $t$-channel states as $\vec{q}_{i}^{\prime}\left(\vec{r}_{i}^{\prime}\right)$ and $\vec{q}_{i}\left(\vec{r}_{i}\right), i=1,2$. At space-time dimension $D=4+2 \epsilon$ the state normalization is

$$
\left\langle\vec{q} \mid \vec{q}^{\prime}\right\rangle=\delta\left(\vec{q}-\vec{q}^{\prime}\right), \quad\left\langle\vec{r} \mid \vec{r}^{\prime}\right\rangle=\delta\left(\vec{r}-\vec{r}^{\prime}\right), \quad\langle\vec{r} \mid \vec{q}\rangle=\frac{e^{i \vec{q} \vec{r}}}{(2 \pi)^{(1+\epsilon)}}
$$

We will also use $\vec{p}_{i j^{\prime}}=\vec{p}_{i}-\vec{p}_{j}^{\prime}$ for brevity.

The $s$-channel discontinuities of scattering amplitudes for the processes $A+B \rightarrow A^{\prime}+B^{\prime}$ have the form

$$
-4 i(2 \pi)^{D-2} \delta\left(\vec{q}_{A}-\vec{q}_{B}\right) \operatorname{disc}_{s} \mathcal{A}_{A B}^{A^{\prime} B^{\prime}}=\left\langle A^{\prime} \bar{A}\left|\left(\frac{s}{s_{0}}\right)^{\hat{\mathcal{K}}} \frac{1}{\hat{\vec{q}}_{1}^{2} \hat{\vec{q}}_{2}^{2}}\right| \bar{B}^{\prime} B\right\rangle .
$$

In this expression $s_{0}$ is an appropriate energy scale, $q_{A}=p_{A^{\prime} A}, \quad q_{B}=p_{B B^{\prime}}$, and $\hat{\mathcal{K}}$ is the BFKL kernel,

$$
\left\langle\vec{q}_{1}, \vec{q}_{2}|\hat{\mathcal{K}}| \vec{q}_{1}^{\prime}, \vec{q}_{2}^{\prime}\right\rangle=\delta\left(\vec{q}_{11^{\prime}}+\vec{q}_{22^{\prime}}\right) \frac{\mathcal{K}_{r}\left(q_{1}, q_{1}^{\prime}, q\right)}{\vec{q}_{1}^{2} \vec{q}_{2}^{2}}+\delta\left(\vec{q}_{22^{\prime}}\right) \delta\left(\vec{q}_{11^{\prime}}\right)\left(\omega\left(\vec{q}_{1}^{2}\right)+\omega\left(\vec{q}_{2}^{2}\right)\right),
$$


where $\omega(t)$ is the gluon Regge trajectory and $\mathcal{K}_{r}\left(q_{1}, q_{1}^{\prime}, q\right)$ represents real particle production in Reggeon collisions. The impact factors are introduced through

$$
\begin{aligned}
& \left\langle\vec{q}_{1}, \vec{q}_{2} \mid \vec{B}^{\prime} B\right\rangle=4 p_{B}^{-} \delta\left(\vec{q}_{B}-\vec{q}_{1}-\vec{q}_{2}\right) \Phi_{B^{\prime} B}\left(\vec{q}_{1}, \vec{q}_{2}\right), \\
& \left\langle A^{\prime} \bar{A} \mid \vec{q}_{1}, \vec{q}_{2}\right\rangle=4 p_{A}^{+} \delta\left(\vec{q}_{A}-\vec{q}_{1}-\vec{q}_{2}\right) \Phi_{A^{\prime} A}\left(\vec{q}_{1}, \vec{q}_{2}\right),
\end{aligned}
$$

where $p^{ \pm}=\left(p_{0} \pm p_{z}\right) / \sqrt{2}$. The kernel $\mathcal{K}_{r}\left(\vec{q}_{1}, \vec{q}_{1}^{\prime} ; \vec{q}\right)$ and the impact factors $\Phi$ are expressed through the Reggeon vertices according to Ref. [27]. So, the real part of the Born kernel reads

$$
\frac{\mathcal{K}_{r}^{B}\left(\vec{q}_{1}, \vec{q}_{1}^{\prime} ; \vec{q}\right)}{\vec{q}_{1}^{2} \vec{q}_{2}^{2}}=\frac{\alpha_{s}\left(\mu^{2}\right) N_{c}}{\pi^{2}}\left(\frac{1}{\vec{q}_{11^{\prime}}^{2}}+\frac{\left(\vec{q}_{2} \vec{q}_{11^{\prime}}\right)}{\vec{q}_{2}^{2} \vec{q}_{11^{\prime}}^{2}}-\frac{\left(\vec{q}_{1} \vec{q}_{11^{\prime}}\right)}{\vec{q}_{1}^{2} \vec{q}_{11^{\prime}}^{2}}-\frac{\left(\overrightarrow{q_{1}} \vec{q}_{2}\right)}{\vec{q}_{1}^{2} \vec{q}_{2}^{2}}\right)
$$

while the one-loop trajectory has the form

$$
\omega\left(\vec{q}^{2}\right)=-\frac{\alpha_{s}\left(\mu^{2}\right) N_{c}}{(2 \pi)^{2+2 \epsilon}} \int d^{2+2 \epsilon} k\left(\frac{2}{\vec{k}^{2}}-\frac{2 \vec{k}(\vec{k}-\vec{q})}{\vec{k}^{2}(\vec{k}-\vec{q})^{2}}\right) .
$$

At once we can see that Eq. (2) does not give the unique definition of the kernel. Indeed, the discontinuity $\operatorname{disc}_{s} \mathcal{A}_{A B}^{A^{\prime} B^{\prime}}$ in Eq. (2) remains intact if one changes both the kernel and the impact factors via an arbitrary nonsingular operator: $\hat{\mathcal{O}}$

$$
\hat{\mathcal{K}} \rightarrow \hat{\mathcal{O}}^{-1} \hat{\mathcal{K}} \hat{\mathcal{O}}, \quad\left\langle A^{\prime} \bar{A}\left|\rightarrow\left\langle A^{\prime} \bar{A}\left|\hat{\mathcal{O}}, \frac{1}{{\hat{\overrightarrow{q_{1}}}}_{1}^{2} \hat{\vec{q}}_{2}^{2}}\right| \bar{B}^{\prime} B\right\rangle \rightarrow \hat{\mathcal{O}}^{-1} \frac{1}{{\hat{\overrightarrow{q_{1}}}}^{2}{\hat{\overrightarrow{q_{2}}}}_{2}^{2}}\right| \bar{B}^{\prime} B\right\rangle .
$$

Actually, the kernel $\hat{\mathcal{K}}(\underline{3})$ is obtained from the symmetric kernel, usually used in the momentum space, just by such transformation. Only owing to this transformation the Möbius form of $\hat{\mathcal{K}}$ is conformal invariant and coincides with the dipole kernel in the leading order. But even if the kernel is fixed in the LO, transformations with $\hat{O}=1-\hat{O}$, where $\hat{O} \sim \alpha_{s}$, are still possible. Within the NLO accuracy these transformations give

$$
\hat{\mathcal{K}} \rightarrow \hat{\mathcal{K}}-\left[\hat{\mathcal{K}}^{B}, \hat{O}\right]
$$

Such transformations can be used for simplify the form of the kernel, in particular of its Möbius form. Indeed, it was shown [10, 21] that this form is simplified by the transformation

$$
\hat{\mathcal{K}} \rightarrow \hat{K}=\hat{\mathcal{K}}+\frac{\alpha_{s}}{8 \pi} \beta_{0}\left[\hat{\mathcal{K}}^{B}, \ln \left(\hat{\vec{q}}_{1}^{2} \hat{\vec{q}}_{2}^{2}\right)\right]
$$

where $\hat{\mathcal{K}}$ is the kernel defined in Eq. (3), $\hat{\mathcal{K}}^{B}=\hat{K}^{B}$ is its LO value and $\beta_{0}$ is the first coefficient of the beta-function. In the following this transformation is assumed to be done. 
In the NLO the Möbius form can be written [10, 21] as follows:

$$
\begin{gathered}
\left\langle\vec{r}_{1}, \vec{r}_{2}\left|\hat{K}_{M}\right| \vec{r}_{1}^{\prime}, \vec{r}_{2}^{\prime}\right\rangle=\frac{\alpha_{s}\left(\mu^{2}\right) N_{c}}{2 \pi^{2}} \int d \vec{\rho} \frac{\vec{r}_{12}^{2}}{\vec{r}_{1 \rho}^{2} \vec{r}_{2 \rho}^{2}}\left[\delta\left(\vec{r}_{11^{\prime}}\right) \delta\left(\vec{r}_{2^{\prime} \rho}\right)+\delta\left(\vec{r}_{1^{\prime} \rho}\right) \delta\left(\vec{r}_{22^{\prime}}\right)-\delta\left(\vec{r}_{11^{\prime}}\right) \delta\left(r_{22^{\prime}}\right)\right] \\
+\frac{\alpha_{s}^{2}\left(\mu^{2}\right) N_{c}^{2}}{4 \pi^{3}}\left[\delta\left(\vec{r}_{11^{\prime}}\right) \delta\left(\vec{r}_{22^{\prime}}\right) \int d \vec{\rho} g_{0}\left(\vec{r}_{1}, \vec{r}_{2} ; \rho\right)+\delta\left(\vec{r}_{11^{\prime}}\right) g_{1}\left(\vec{r}_{1}, \vec{r}_{2} ; \vec{r}_{2}^{\prime}\right)+\delta\left(\vec{r}_{22^{\prime}}\right) g_{1}\left(\vec{r}_{2}, \vec{r}_{1} ; \vec{r}_{1}^{\prime}\right)\right. \\
\left.+\frac{1}{\pi} g_{2}\left(\vec{r}_{1}, \vec{r}_{2} ; \vec{r}_{1}^{\prime}, \vec{r}_{2}^{\prime}\right)\right] .
\end{gathered}
$$

Here $\vec{r}_{i \rho}=\vec{r}_{i}-\vec{\rho}$, and the whole kernel is symmetric with respect to the substitution $1 \leftrightarrow 2,1^{\prime} \leftrightarrow$ $2^{\prime}$. The Möbius kernel (11) is defined with an accuracy to any functions independent of $\vec{r}_{1}$ or of $\vec{r}_{2}$ such that after their addition the kernel remains zero at $\vec{r}_{1}=\vec{r}_{2}$ [10, 21]. Therefore, one can add to the kernel only the functions which are antisymmetric with respect to the $\vec{r}_{1} \leftrightarrow \vec{r}_{2}$ substitution. These functions do not change the symmetric part of the kernel. But this part alone plays a role because of the symmetry of the impact factors.

The transformation (10) considerably simplifies the "non-abelian" piece of the quark part of the Möbius form [10]. In particular, it removes its contribution to the function $g_{2}$. Moreover, just after this transformation the Möbius form of the quark contribution to the BFKL kernel [10, 18] coincides with the quark part of the linearized BK kernel [15, 16] calculated in the colour dipole model.

However, the transformation (10) does not remove the disagreement of the gluon contribution. The BFKL framework gives for the gluon contribution to the functions $g_{i}$ in (11) [21]

$$
\begin{gathered}
g_{0}\left(\vec{r}_{1}, \vec{r}_{2} ; \vec{\rho}\right)=2 \pi \zeta(3) \delta(\vec{\rho})-g\left(\vec{r}_{1}, \vec{r}_{2} ; \vec{\rho}\right), \\
g_{1}\left(\vec{r}_{1}, \vec{r}_{2} ; \vec{r}_{2}^{\prime}\right)=\frac{11}{6} \frac{\vec{r}_{12}^{2}}{\vec{r}_{22^{\prime}}^{2} \vec{r}_{12^{\prime}}^{2}} \ln \left(\frac{\vec{r}_{12}^{2}}{r_{\mu}^{2}}\right)+\frac{11}{6}\left(\frac{1}{\vec{r}_{22^{\prime}}^{2}}-\frac{1}{\vec{r}_{12^{\prime}}^{2}}\right) \ln \left(\frac{\vec{r}_{22^{\prime}}^{2}}{\vec{r}_{12^{\prime}}^{2}}\right) \\
+\frac{1}{2 \vec{r}_{22^{\prime}}^{2}} \ln \left(\frac{\vec{r}_{12^{\prime}}^{2}}{\vec{r}_{22^{\prime}}^{2}}\right) \ln \left(\frac{\vec{r}_{12}^{2}}{\vec{r}_{12^{\prime}}^{2}}\right)-\frac{\vec{r}_{12}^{2}}{2 \vec{r}_{22^{\prime}}^{2} \vec{r}_{12^{\prime}}^{2}} \ln \left(\frac{\vec{r}_{12}^{2}}{\vec{r}_{22^{\prime}}^{2}}\right) \ln \left(\frac{\vec{r}_{12}^{2}}{\vec{r}_{12^{\prime}}^{2}}\right),
\end{gathered}
$$

where

$$
\ln r_{\mu}^{2}=2 \psi(1)-\ln \frac{\mu^{2}}{4}-\frac{3}{11}\left(\frac{67}{9}-2 \zeta(2)\right)
$$


and

$$
\begin{aligned}
& g_{2}\left(\vec{r}_{1}, \vec{r}_{2} ; \vec{r}_{1}^{\prime}, \vec{r}_{2}^{\prime}\right)=\frac{1}{2 \vec{r}_{1^{\prime} 2^{\prime}}^{4}}\left(\frac{\vec{r}_{11^{\prime}}^{2} \vec{r}_{22^{\prime}}^{2}-2 \vec{r}_{12}^{2} \vec{r}_{1^{\prime} 2^{\prime}}^{2}}{d} \ln \left(\frac{\vec{r}_{12^{\prime}}^{2} \vec{r}_{21^{\prime}}^{2}}{\vec{r}_{11^{\prime}}^{2} \vec{r}_{22^{\prime}}^{2}}\right)-1\right)+\frac{\vec{r}_{12}^{2} \ln \left(\frac{\vec{r}_{11^{\prime}}^{2}}{\vec{r}_{1^{\prime} 2^{\prime}}^{2}}\right)}{2 \vec{r}_{11^{\prime}}^{2} \vec{r}_{12^{\prime}}^{2} \vec{r}_{22^{\prime}}^{2}} \\
& +\frac{\ln \left(\frac{\vec{r}_{12^{\prime}}^{2} \vec{r}_{21^{\prime}}^{2}}{\vec{r}_{11^{\prime}}^{2} \vec{r}_{22^{\prime}}^{2}}\right)}{4 \vec{r}_{11^{\prime}}^{2} \vec{r}_{22^{\prime}}^{2}}\left(\frac{\vec{r}_{12}^{4}}{d}-\frac{\vec{r}_{12}^{2}}{\vec{r}_{11^{\prime} 2^{\prime}}^{2}}\right)+\frac{\ln \left(\frac{\vec{r}_{12}^{2} \vec{r}_{1^{\prime} \prime^{\prime}}^{2}}{\vec{r}_{11^{\prime}}^{2} \vec{r}_{22^{\prime}}^{2}}\right)}{2 \vec{r}_{12^{\prime}}^{2} \vec{r}_{21^{\prime}}^{2}}\left(\frac{\vec{r}_{12}^{2}}{2 \vec{r}_{11^{\prime} 2^{\prime}}^{2}}+\frac{1}{2}-\frac{\vec{r}_{22^{\prime}}^{2}}{\vec{r}_{1^{\prime} 2^{\prime}}^{2}}\right) \\
& +\frac{\vec{r}_{21^{\prime}}^{2} \ln \left(\frac{\vec{r}_{21^{\prime}}^{2} \vec{r}_{1^{\prime}{ }^{\prime}}^{2}}{\vec{r}_{12}^{2} \vec{r}_{11^{\prime}}^{\prime}}\right)}{2 \vec{r}_{11^{\prime}}^{2} \vec{r}_{22^{\prime}}^{2} \vec{r}_{1^{\prime} 2^{\prime}}^{2}}+\frac{\ln \left(\frac{\vec{r}_{12}^{2}}{\vec{r}_{12^{\prime}}^{2}}\right)}{4 \vec{r}_{11^{\prime}}^{2} \vec{r}_{22^{\prime}}^{2}}+\frac{\ln \left(\frac{\vec{r}_{22^{\prime}}^{2}}{\vec{r}_{12}^{2}}\right)}{2 \vec{r}_{11^{\prime}}^{2} \vec{r}_{12^{\prime}}^{2}}+\frac{\vec{r}_{12}^{2} \ln \left(\frac{\vec{r}_{12}^{2} \vec{r}_{1^{\prime}}^{2}}{\vec{r}_{12^{\prime}}^{2} \vec{r}_{21^{\prime}}^{2}}\right)}{4 \vec{r}_{11^{\prime}}^{2} \vec{r}_{22^{\prime}}^{2} \vec{r}_{12^{\prime} 2^{\prime}}^{2}} \\
& +\frac{\ln \left(\frac{\vec{r}_{12}^{2} \vec{r}_{12^{\prime}}^{2}}{\vec{r}_{12^{\prime}}^{2} \vec{r}_{22^{\prime}}^{2}}\right)}{2 \vec{r}_{11^{\prime}}^{2} \vec{r}_{1^{\prime} 2^{\prime}}^{2}}+\frac{\ln \left(\frac{\vec{r}_{12}^{2} \vec{r}_{11^{\prime}}^{2}}{\vec{r}_{22^{\prime}}^{2} \vec{r}_{1^{\prime} 2^{\prime}}^{2}}\right)}{2 \vec{r}_{12^{\prime}}^{2} \vec{r}_{1^{\prime} 2^{\prime}}^{2}}+\left(1 \leftrightarrow 2,1^{\prime} \leftrightarrow 2^{\prime}\right), \quad d=\vec{r}_{12^{\prime}}^{2} \vec{r}_{21^{\prime}}^{2}-\vec{r}_{11^{\prime}}^{2} \vec{r}_{22^{\prime}}^{2} .
\end{aligned}
$$

Remind that the coefficients of $\delta\left(\vec{r}_{11^{\prime}}\right) \delta\left(r_{22^{\prime}}\right)$ in Eq. (11) are written in the integral form in order to make explicit cancellation of the ultraviolet singularities of separate terms. Therefore one can take $g_{0}$ in various forms (without change of the integral $\int d \vec{\rho} g_{0}\left(\vec{r}_{1}, \vec{r}_{2} ; \rho\right)$ ). Here we change the form of $g_{0}$ in comparison with our previous papers using the equalities

$$
\int d \vec{\rho} \frac{\vec{r}_{12}^{2}}{\vec{r}_{1 \rho}^{2} \vec{r}_{2 \rho}^{2}} \ln \left(\frac{\vec{r}_{1 \rho}^{2}}{\vec{r}_{12}^{2}}\right) \ln \left(\frac{\vec{r}_{2 \rho}^{2}}{\vec{r}_{12}^{2}}\right)=\int \frac{d \vec{\rho}}{\vec{r}_{2 \rho}^{2}} \ln \left(\frac{\vec{r}_{1 \rho}^{2}}{\vec{r}_{12}^{2}}\right) \ln \left(\frac{\vec{r}_{1 \rho}^{2}}{\vec{r}_{2 \rho}^{2}}\right)=4 \pi \zeta(3) .
$$

In the colour dipole approach the gluon contribution was found in Ref. [17]. With account of the correction given in Ref. [25], it gives

$$
\begin{gathered}
g_{0}^{B C}\left(\vec{r}_{1}, \vec{r}_{2}, \vec{\rho}\right)=2 \pi \zeta(3) \delta(\vec{\rho})-g_{1}^{B C}\left(\vec{r}_{1}, \vec{r}_{2}, \vec{\rho}\right), \\
g_{1}^{B C}\left(\vec{r}_{1}, \vec{r}_{2} ; \vec{r}_{2}^{\prime}\right)=\frac{11}{6} \frac{\vec{r}_{12}^{2}}{\vec{r}_{22^{\prime}}^{2} \vec{r}_{12^{\prime}}^{2}} \ln \left(\frac{\vec{r}_{12}^{2}}{r_{\mu_{B C}}^{2}}\right)+\frac{11}{6}\left(\frac{1}{\vec{r}_{22^{\prime}}^{2}}-\frac{1}{\vec{r}_{12^{\prime}}^{2}}\right) \ln \left(\frac{\vec{r}_{22^{\prime}}^{2}}{\vec{r}_{12^{\prime}}^{2}}\right) \\
-\frac{\vec{r}_{12}^{2}}{\vec{r}_{22^{\prime}}^{2} \vec{r}_{12^{\prime}}} \ln \left(\frac{\vec{r}_{12}^{2}}{\vec{r}_{22^{\prime}}}\right) \ln \left(\frac{\vec{r}_{12}^{2}}{\vec{r}_{12^{\prime}}}\right),
\end{gathered}
$$

where

$$
\begin{gathered}
\ln r_{\mu_{B C}}^{2}=-\ln \mu^{2}-\frac{3}{11}\left(\frac{67}{9}-2 \zeta(2)\right), \\
g_{2}^{B C}\left(\vec{r}_{1}, \vec{r}_{2} ; \vec{r}_{1}^{\prime}, \vec{r}_{2}^{\prime}\right)=\ln \left(\frac{\vec{r}_{12^{\prime}}^{2} \vec{r}_{21^{\prime}}^{2}}{\vec{r}_{11^{\prime}}^{2} \vec{r}_{22^{\prime}}^{2}}\right)\left[\frac{\vec{r}_{11^{\prime}}^{2} \vec{r}_{22^{\prime}}^{2}+\vec{r}_{12^{\prime}}^{2} \vec{r}_{21^{\prime}}^{2}-4 \vec{r}_{12}^{2} \vec{r}_{1^{\prime} 2^{\prime}}^{2}}{2 d \vec{r}_{1^{\prime} 2^{\prime}}^{4}}\right. \\
\left.+\frac{1}{4 \vec{r}_{11^{\prime}}^{2} \vec{r}_{22^{\prime}}^{2}}\left(\frac{\vec{r}_{12}^{4}}{d}-\frac{\vec{r}_{12}^{2}}{\vec{r}_{1^{\prime} 2^{\prime}}^{2}}\right)+\frac{1}{4 \vec{r}_{12^{\prime}}^{2} \vec{r}_{21^{\prime}}^{2}}\left(\frac{\vec{r}_{12}^{4}}{d}+\frac{\vec{r}_{12}^{2}}{\vec{r}_{1^{\prime} 2^{\prime}}^{2}}\right)\right]-\frac{1}{\vec{r}_{1^{\prime} 2^{\prime}}^{4}} .
\end{gathered}
$$




\section{Matching of the gluon parts}

In principle, if the kernels $\hat{K}_{M}$ and $\hat{\mathcal{K}}_{B C}$ can be connected by the transformation (9), one can easily write a formal expression for the operator $\hat{O}$. Indeed, let us denote $\hat{K}_{M}-\hat{\mathcal{K}}_{B C}=\hat{\Delta}$, the eigenstates of the Born kernel $\hat{\mathcal{K}}^{B}|\mu\rangle$, and the corresponding eigenvalues $\omega_{\mu}^{B}$. Then, if $\hat{\Delta}=\left[\hat{\mathcal{K}}^{B}, \hat{O}\right]$, one has

$$
\left(\omega_{\mu^{\prime}}^{B}-\omega_{\mu}^{B}\right)\left\langle\mu^{\prime}|\hat{O}| \mu\right\rangle=\left\langle\mu^{\prime}|\hat{\Delta}| \mu\right\rangle .
$$

It can be seen from this equation that the operator $\hat{O}$ exists only if the operator $\hat{\Delta}$ has zero matrix elements between states of equal eigenvalues. If so, supposing that the states $|\mu\rangle$ form a complete set, one has

$$
\hat{O}=\sum_{\mu, \mu^{\prime}} \frac{\left|\mu^{\prime}\right\rangle\left\langle\mu^{\prime}|\hat{\Delta}| \mu\right\rangle\langle\mu|}{\omega_{\mu^{\prime}}^{B}-\omega_{\mu}^{B}}
$$

and

$$
\left\langle\vec{r}_{1}, \vec{r}_{2}|\hat{O}| \vec{r}_{1}^{\prime}, \vec{r}_{2}^{\prime}\right\rangle=\sum_{\mu, \mu^{\prime}} \frac{\left\langle\vec{r}_{1}, \vec{r}_{2} \mid \mu^{\prime}\right\rangle\left\langle\mu^{\prime}|\hat{\Delta}| \mu\right\rangle\left\langle\mu \mid \vec{r}_{1}^{\prime}, \vec{r}_{2}^{\prime}\right\rangle}{\omega_{\mu^{\prime}}^{B}-\omega_{\mu}^{B}} .
$$

Since we know $\left\langle\vec{r}_{1}, \vec{r}_{2}|\hat{\Delta}| \vec{r}_{1}^{\prime}, \vec{r}_{2}^{\prime}\right\rangle$ from Eqs. (11), (12)-(15) and(17)-(20), we can find

$$
\left\langle\mu^{\prime}|\hat{\Delta}| \mu\right\rangle=\int d \vec{r}_{1} d \vec{r}_{2} d \vec{r}_{1}^{\prime} d \vec{r}_{2}^{\prime}\left\langle\mu^{\prime} \mid \vec{r}_{1}^{\prime}, \vec{r}_{2}^{\prime}\right\rangle\left\langle\vec{r}_{1}^{\prime}, \vec{r}_{2}^{\prime}|\hat{\Delta}| \vec{r}_{1}, \vec{r}_{2}\right\rangle\left\langle\vec{r}_{1}, \vec{r}_{2} \mid \mu\right\rangle
$$

using the known eigenfunctions $\left\langle\vec{r}_{1}, \vec{r}_{2} \mid \mu\right\rangle$ in the coordinate space [8] and then $\left\langle\vec{r}_{1}, \vec{r}_{2}|\hat{O}| \vec{r}_{1}^{\prime}, \vec{r}_{2}^{\prime}\right\rangle$ using Eq. (23) . However, it is rather difficult because of complexity of the eigenfunctions and the corresponding eigenvalues. In fact, we did not do it, but we have guessed the operator $\hat{O}$ :

$$
\begin{gathered}
\left\langle\vec{q}_{1}, \overrightarrow{q_{2}}|\hat{O}| \vec{q}_{1}^{\prime}, \vec{q}_{2}^{\prime}\right\rangle=-\delta\left(\vec{q}_{11^{\prime}}+\vec{q}_{22^{\prime}}\right) \frac{\mathcal{K}_{r}^{B}\left(\vec{q}_{1}, \vec{q}_{1}^{\prime} ; \vec{q}\right)}{2 \vec{q}_{1}^{2} \vec{q}_{2}^{2}} \ln \vec{q}_{11^{\prime}}^{2} \\
+\frac{\alpha_{s} N_{c}}{4 \pi^{2}} \delta\left(\vec{q}_{22^{\prime}}\right) \delta\left(\vec{q}_{11^{\prime}}\right) \int d^{2+2 \epsilon} k \ln \vec{k}^{2}\left(\frac{2}{\vec{k}^{2}}-\frac{\vec{k}\left(\vec{k}-\vec{q}_{1}\right)}{\vec{k}^{2}\left(\vec{k}-\vec{q}_{1}\right)^{2}}-\frac{\vec{k}\left(\vec{k}-\vec{q}_{2}\right)}{\vec{k}^{2}\left(\vec{k}-\vec{q}_{2}\right)^{2}}\right) .
\end{gathered}
$$

Let us show that the transformation defined by Eqs. (9) and (25), being applied to the kernel $\hat{K}_{M}$, converts the functions $g_{i}$ given by Eqs. (11) and (12)-(15) into the functions $g_{i}^{B C}$ (17)-(20).

In the momentum space, we have for the commutator $\left[\hat{\mathcal{K}}^{B}, \hat{O}\right]$

$$
\left\langle\vec{q}_{1}, \vec{q}_{2}\left|\left[\hat{\mathcal{K}}^{B}, \hat{O}\right]\right| \vec{q}_{1}^{\prime}, \vec{q}_{2}^{\prime}\right\rangle=\delta\left(\vec{q}_{11^{\prime}}+\vec{q}_{22^{\prime}}\right)\left[\int d \vec{k} \frac{\mathcal{K}_{r}^{B}\left(\vec{q}_{1}, \vec{q}_{1}-\vec{k} ; \vec{q}\right)}{2 \vec{q}_{1}^{2} \vec{q}_{2}^{2}} \frac{\mathcal{K}_{r}^{B}\left(\vec{q}_{1}-\vec{k}, \vec{q}_{1}^{\prime} ; \vec{q}\right)}{\left(\vec{k}-\vec{q}_{1}\right)^{2}\left(\vec{k}+\overrightarrow{q_{2}}\right)^{2}} \ln \frac{\vec{k}^{2}}{\left(\vec{k}-\vec{q}_{11^{\prime}}\right)^{2}}\right.
$$




$$
\left.+\frac{\alpha_{s} N_{c}}{4 \pi^{2}} \frac{\mathcal{K}_{r}^{B}\left(\vec{q}_{1}, \vec{q}_{1}^{\prime} ; \vec{q}\right)}{\vec{q}_{1}^{2} \vec{q}_{2}^{2}} \int d \vec{k}\left(\frac{\vec{k}\left(\vec{k}-\vec{q}_{1}^{\prime}\right)}{\vec{k}^{2}\left(\vec{k}-\vec{q}_{1}^{\prime}\right)^{2}}-\frac{\vec{k}\left(\vec{k}-\vec{q}_{1}\right)}{\vec{k}^{2}\left(\vec{k}-\vec{q}_{1}\right)^{2}}+\left(1 \leftrightarrow 2,1^{\prime} \leftrightarrow 2^{\prime}\right)\right) \ln \frac{\vec{q}_{11^{\prime}}^{2}}{\vec{k}^{2}}\right] .
$$

Since all integrals here and below are convergent, we put $\epsilon=0$ henceforth. The first line in Eq. (26) is equal to the doubled contribution $\left\langle\vec{q}_{1}, \vec{q}_{2}\left|\hat{\mathcal{K}}_{s 2}\right| \vec{q}_{1}^{\prime}, \vec{q}_{2}^{\prime}\right\rangle$ to the BFKL kernel, defined in Ref. [21]. Denoting the remaining terms in Eq. (26) as $\left\langle\vec{q}_{1}, \vec{q}_{2}|\hat{\mathcal{V}}| \vec{q}_{1}^{\prime}, \vec{q}_{2}^{\prime}\right\rangle$, after integration we obtain

$$
\begin{aligned}
\left\langle\vec{q}_{1}, \vec{q}_{2}|\hat{\mathcal{V}}| \vec{q}_{1}^{\prime}, \vec{q}_{2}^{\prime}\right\rangle & =\delta\left(\vec{q}_{11^{\prime}}+\vec{q}_{22^{\prime}}\right) \frac{\left(\alpha_{s} N_{c}\right)^{2}}{8 \pi^{3}}\left(\frac{1}{\vec{k}^{2}}+\frac{\left(\overrightarrow{q_{2}} \vec{k}\right)}{\vec{q}_{2}^{2} \vec{k}^{2}}-\frac{\left(\overrightarrow{q_{1}} \vec{k}\right)}{\vec{q}_{1}^{2} \vec{k}^{2}}-\frac{\left(\vec{q}_{1} \vec{q}_{2}\right)}{\vec{q}_{1}^{2} \vec{q}_{2}^{2}}\right) \\
& \times\left(\ln ^{2} \frac{\vec{q}_{1}^{\prime 2}}{\vec{k}^{2}}+\ln ^{2} \frac{\vec{q}_{2}^{\prime 2}}{\vec{k}^{2}}-\ln ^{2} \frac{\vec{q}_{1}^{2}}{\vec{k}^{2}}-\ln ^{2} \frac{\vec{q}_{2}^{2}}{\vec{k}^{2}}\right),
\end{aligned}
$$

where $\vec{k}=\vec{q}_{11^{\prime}}$.

The Möbius form of $\hat{\mathcal{K}}_{s 2}$ was found in Ref. 21]. To obtain such form for the operator $\hat{\mathcal{V}}$ we have to transform Eq. (27) into the coordinate space. It can be done using the following integrals:

$$
\begin{gathered}
\int \frac{d \vec{k}}{2 \pi} e^{i \vec{k} \vec{r}} \frac{\vec{k}}{\vec{k}^{2}}=\frac{i \vec{r}}{\vec{r}^{2}}, \\
\int \frac{d \vec{q}}{2 \pi} \int \frac{d \vec{k}}{2 \pi} e^{i[\vec{q} \vec{r}+\vec{k} \vec{\rho}]} \frac{1}{\vec{q}^{2}} \ln \frac{(\vec{q}-\vec{k})^{2}}{\vec{k}^{2}} \ln \frac{(\vec{q}-\vec{k})^{2}}{\vec{q}^{2}}=\frac{1}{\vec{\rho}^{2}} \ln \left(\frac{(\vec{r}+\vec{\rho})^{2}}{\vec{r}^{2}}\right) \ln \left(\frac{(\vec{r}+\vec{\rho})^{2}}{\vec{\rho}^{2}}\right), \\
\int \frac{d \vec{q}}{2 \pi} \int \frac{d \vec{k}}{2 \pi} e^{i[\vec{q} \vec{r}+\vec{k} \vec{\rho}]} \frac{1}{\vec{q}^{2}} \ln ^{2} \frac{(\vec{q}-\vec{k})^{2}}{\vec{k}^{2}}=\frac{1}{\vec{\rho}^{2}} \ln ^{2}\left(\frac{(\vec{r}+\vec{\rho})^{2}}{\vec{r}^{2}}\right), \\
\int \frac{d \vec{q}}{2 \pi} \int \frac{d \vec{k}}{2 \pi} e^{i[\vec{q} \vec{r}+\vec{k} \vec{\rho}]} \frac{(\vec{q} \vec{k})}{\vec{q}^{2} \vec{k}{ }^{2}} \ln ^{2} \frac{\left(\vec{k}+\vec{q}^{2}\right.}{\vec{q}^{2}}=-\frac{(\vec{r} \vec{\rho})}{\vec{r}^{2} \vec{\rho}^{2}} \ln ^{2}\left(\frac{(\vec{\rho}-\vec{r})^{2}}{\vec{\rho}^{2}}\right), \\
\int \frac{d \vec{q}}{2 \pi} \int \frac{d \vec{k}}{2 \pi} e^{i[\vec{q} \vec{r}+\vec{k} \vec{\rho}]} \frac{(\vec{q} \vec{k})}{\vec{q}^{2} \vec{k}^{2}} \ln ^{2} \frac{\vec{k}^{2}}{\vec{q}^{2}}=-\frac{(\vec{r} \vec{\rho})}{\vec{r}^{2} \vec{\rho}^{2}} \ln ^{2}\left(\frac{\vec{\rho}^{2}}{\vec{r}^{2}}\right), \\
\int \frac{d \overrightarrow{q_{1}}}{2 \pi} \int \frac{d \overrightarrow{q_{2}}}{2 \pi} \int \frac{d \vec{k}}{2 \pi} e^{\left.i \vec{q} \vec{q}_{1} \vec{r}_{1}+\vec{q}_{2} \vec{r}_{2}+\vec{k} \vec{\rho}\right]} \frac{\left(\vec{q}_{1} \vec{q}_{2}\right)}{\vec{q}_{1}^{2} \vec{q}_{2}^{2}} \ln ^{2} \frac{\vec{q}_{1}^{2}}{\vec{k}^{2}}=\frac{4\left(\vec{r}_{1} \vec{r}_{2}\right)}{\vec{r}_{1}^{2} \vec{r}_{2}^{2} \vec{\rho}^{2}} \ln \left(\frac{\vec{r}_{1}^{2}}{\vec{\rho}^{2}}\right) .
\end{gathered}
$$

The result is

$$
\begin{gathered}
\frac{8 \pi^{4}}{\left(\alpha_{s} N_{c}\right)^{2}}\left\langle\vec{r}_{1} \vec{r}_{2}|\hat{\mathcal{V}}| \vec{r}_{1}^{\prime} \vec{r}_{2}^{\prime}\right\rangle=v_{M}\left(\vec{r}_{1}, \vec{r}_{2} ; \vec{r}_{1}^{\prime}, \vec{r}_{2}^{\prime}\right) \\
+\left[\frac{\vec{r}_{11^{\prime}}^{2}-\vec{r}_{1^{\prime} 2^{\prime}}^{2}}{\vec{r}_{11^{\prime}}^{2} \vec{r}_{12^{\prime}}^{2} \vec{r}_{1^{\prime} 2^{\prime}}^{2}} \ln \left(\frac{\vec{r}_{11^{\prime}}^{2}}{\vec{r}_{12^{\prime}}^{2}}\right)+\frac{1}{\vec{r}_{11^{\prime}}^{2} \vec{r}_{1^{\prime} 2^{\prime}}^{2}} \ln \left(\frac{\vec{r}_{11^{\prime}}^{2} \vec{r}_{12^{\prime}}^{2}}{\vec{r}_{1^{\prime} 2^{\prime}}^{4}}\right)+\left(1 \leftrightarrow 2,1^{\prime} \leftrightarrow 2^{\prime}\right)\right],
\end{gathered}
$$


where

$$
\begin{gathered}
v_{M}\left(\vec{r}_{1}, \vec{r}_{2} ; \vec{r}_{1}^{\prime}, \vec{r}_{2}^{\prime}\right)=\pi \delta\left(\vec{r}_{22^{\prime}}\right) \frac{\vec{r}_{12}^{2}+\vec{r}_{21^{\prime}}^{2}-\vec{r}_{11^{\prime}}^{2}}{2 \vec{r}_{11^{\prime}}^{2} \vec{r}_{21^{\prime}}^{2}} \ln \left(\frac{\vec{r}_{21^{\prime}}^{2}}{\vec{r}_{12}^{2}}\right) \ln \left(\frac{\vec{r}_{11^{\prime}}^{4}}{\vec{r}_{12}^{2} \vec{r}_{21^{\prime}}^{2}}\right) \\
-\vec{r}_{12}^{2}\left(\frac{\ln \left(\frac{\vec{r}_{12^{\prime}}^{2}}{\vec{r}_{11^{\prime}}^{2}}\right)}{\vec{r}_{11^{\prime}}^{2} \vec{r}_{12^{\prime}}^{2} \vec{r}_{22^{\prime}}^{2}}+\frac{\ln \left(\frac{\vec{r}_{22^{\prime}}^{2}}{\vec{r}_{21^{\prime}}^{2}}\right)}{\vec{r}_{11^{\prime}}^{2} \vec{r}_{21^{\prime}}^{2} \vec{r}_{1^{\prime} 2^{\prime}}^{2}}+\frac{\ln \left(\frac{\vec{r}_{12^{\prime}}^{2}}{\vec{r}_{1^{\prime}}^{2}}\right)}{\vec{r}_{11^{\prime}}^{2}, \vec{r}_{22^{\prime}}^{2} \vec{r}_{1^{\prime} 2^{\prime}}^{2}}\right) \\
-\frac{\vec{r}_{22^{\prime}}^{2}-\vec{r}_{12^{\prime}}^{2}}{\vec{r}_{11^{\prime}}^{2} \vec{r}_{22^{\prime}}^{2} \vec{r}_{1^{\prime} 2^{\prime}}^{2}} \ln \left(\frac{\vec{r}_{12^{\prime}}^{2} \vec{r}_{21^{\prime}}^{2}}{\vec{r}_{22^{\prime}}^{2} \vec{r}_{1^{\prime} 2^{\prime}}^{2}}\right)+\left(1 \leftrightarrow 2,1^{\prime} \leftrightarrow 2^{\prime}\right) .
\end{gathered}
$$

The terms in the square brackets in Eq. (34) do not depend either on $\vec{r}_{1}$ or on $\vec{r}_{2}$ and therefore they are omitted in the Möbius form. As for $v_{M}\left(\vec{r}_{1}, \vec{r}_{2} ; \vec{r}_{1}^{\prime}, \vec{r}_{2}^{\prime}\right)$, it turns into zero at $\vec{r}_{1}=\vec{r}_{2}$, so that it satisfies the requirements for the Möbius forms. Using the Möbius form of $\hat{\mathcal{K}}_{s 2}$ [21] (see Eqs. (44), (54), (55), (67) and (69) there) and Eq. (35), we obtain

$$
\begin{aligned}
& \frac{8 \pi^{4}}{\left(\alpha_{s} N_{c}\right)^{2}}\left\langle\vec{r}_{1} \vec{r}_{2}\left|\left[\hat{\mathcal{K}}^{B}, \hat{O}\right]_{M}\right| \vec{r}_{1}^{\prime} \vec{r}_{2}^{\prime}\right\rangle=\pi \delta\left(\vec{r}_{22^{\prime}}\right)\left[\frac{1}{\vec{r}_{11^{\prime}}^{2}} \ln \left(\frac{\vec{r}_{21^{\prime}}^{2}}{\vec{r}_{12}^{2}}\right) \ln \left(\frac{\vec{r}_{11^{\prime}}^{2}}{\vec{r}_{21^{\prime}}^{2}}\right)-\frac{\vec{r}_{12}^{2}}{\vec{r}_{11^{\prime}}^{2} \vec{r}_{21^{\prime}}^{2}} \ln \left(\frac{\vec{r}_{11^{\prime}}^{2}}{\vec{r}_{12}^{2}}\right) \ln \left(\frac{\vec{r}_{12}^{2}}{\vec{r}_{21^{\prime}}^{2}}\right)\right] \\
& -\vec{r}_{12}^{2}\left(\frac{\ln \left(\frac{\vec{r}_{121^{\prime}}^{2} \vec{r}_{21^{\prime}}^{2}}{\vec{r}_{12}^{2} \vec{r}_{1^{\prime} 2^{\prime}}^{2}}\right)}{2 \vec{r}_{11^{\prime}}^{2} \vec{r}_{22^{\prime}}^{2} \vec{r}_{1^{\prime} 2^{\prime}}^{2}}+\frac{\ln \left(\frac{\vec{r}_{1^{\prime} 2^{\prime}}^{2}}{\vec{r}_{22^{\prime}}^{2}}\right)}{\vec{r}_{11^{\prime}}^{2} \vec{r}_{21^{\prime}}^{2} \vec{r}_{22^{\prime}}^{2}}+\frac{\ln \left(\frac{\vec{r}_{11^{\prime}}^{2} \vec{r}_{22^{\prime}}^{2}}{\vec{r}_{12}^{2} \vec{r}_{1^{\prime} 2^{\prime}}^{2}}\right)}{2 \vec{r}_{12^{\prime}}^{2} \vec{r}_{21^{\prime}}^{2} \vec{r}_{1^{\prime} 2^{\prime}}^{2}}\right)-\frac{\ln \left(\frac{\vec{r}_{22^{\prime}}^{2} \vec{r}_{1^{\prime} 2^{\prime}}^{2}}{\vec{r}_{12}^{2} \vec{r}_{12^{\prime}}^{2}}\right)}{\vec{r}_{12^{\prime}}^{2} \vec{r}_{1^{\prime} 2^{\prime}}^{2}}-\frac{\ln \left(\frac{\vec{r}_{12}^{2} \vec{r}_{11^{\prime}}^{2}}{\vec{r}_{21^{\prime}}^{2} \vec{r}_{1^{\prime} 2^{\prime}}^{2}}\right) \vec{r}_{21^{\prime}}^{2}}{\vec{r}_{11^{\prime}}^{2}, \vec{r}_{22^{\prime}}^{2} \vec{r}_{1^{\prime} 2^{\prime}}^{2}} \\
& -\frac{\ln \left(\frac{\vec{r}_{12}^{2} \vec{r}_{12^{\prime}}^{2}}{\vec{r}_{11^{\prime}}^{2} \vec{r}_{22^{\prime}}^{2}}\right)}{\vec{r}_{11^{\prime}}^{2} \vec{r}_{12^{\prime}}^{2}}-\frac{\ln \left(\frac{\vec{r}_{1^{\prime} 2^{\prime}}^{2}}{\vec{r}_{12}^{2}}\right)}{2 \vec{r}_{11^{\prime}}^{2} \vec{r}_{22^{\prime}}^{2}}-\frac{\ln \left(\frac{\vec{r}_{11^{\prime}}^{2} \vec{r}_{22^{\prime}}^{2}}{\vec{r}_{12}^{2} \vec{r}_{1^{\prime} 2^{\prime}}^{2}}\right)}{2 \vec{r}_{12^{\prime}}^{2} \vec{r}_{21^{\prime}}^{2}}-\frac{\ln \left(\frac{\vec{r}_{12}^{2} \vec{r}^{2}}{\vec{r}_{11^{\prime} 2^{\prime}}^{2} \vec{r}_{22^{\prime}}^{2}}\right) \vec{r}_{22^{\prime}}^{2}}{\vec{r}_{12^{\prime}}^{2} \vec{r}_{21^{\prime}}^{2} \vec{r}_{1^{\prime} 2^{\prime}}^{2}}-\frac{\ln \left(\frac{\vec{r}_{11^{\prime}}^{2} \vec{r}_{22^{\prime}}^{2}}{\vec{r}_{12}^{2} \vec{r}_{1^{\prime} 2^{\prime}}^{2}}\right)}{\vec{r}_{11^{\prime}}^{2} \vec{r}_{1^{\prime} 2^{\prime}}^{2}}+\left(1 \leftrightarrow 2,1^{\prime} \leftrightarrow 2^{\prime}\right) .
\end{aligned}
$$

¿From the definition (11) it follows that the transformation $\hat{K}_{M} \rightarrow \hat{\mathcal{K}}_{M}-\left[\hat{\mathcal{K}}^{B}, \hat{O}\right]_{M}$ leaves $g_{0}$ untouched and changes only $g_{1,2}$. Using Eqs. (13), (15) we get

$$
\begin{aligned}
g_{1}\left(\vec{r}_{1}, \vec{r}_{2} ; \vec{r}_{2}^{\prime}\right) \rightarrow g_{1}^{T}\left(\vec{r}_{1}, \vec{r}_{2} ; \vec{r}_{2}^{\prime}\right)=\frac{11}{6} \frac{\vec{r}_{12}^{2}}{\vec{r}_{22^{\prime}}^{2} \vec{r}_{12^{\prime}}^{2}} \ln \left(\frac{\vec{r}_{12}^{2}}{r_{\mu}^{2}}\right)+\frac{11}{6}\left(\frac{1}{\vec{r}_{22^{\prime}}^{2}}-\frac{1}{\vec{r}_{12^{\prime}}^{2}}\right) \ln \left(\frac{\vec{r}_{22^{\prime}}^{2}}{\vec{r}_{12^{\prime}}^{2}}\right) \\
-\frac{\vec{r}_{12}^{2}}{\vec{r}_{22^{\prime}}^{2} \vec{r}_{12^{\prime}}^{2}} \ln \left(\frac{\vec{r}_{12}^{2}}{\vec{r}_{22^{\prime}}^{2}}\right) \ln \left(\frac{\vec{r}_{12}^{2}}{\vec{r}_{12^{\prime}}^{2}}\right),
\end{aligned}
$$

and

$$
\begin{aligned}
& g_{2}\left(\vec{r}_{1}, \vec{r}_{2} ; \vec{r}_{1}^{\prime}, \vec{r}_{2}^{\prime}\right) \rightarrow g_{2}^{T}\left(\vec{r}_{1}, \vec{r}_{2} ; \vec{r}_{1}^{\prime}, \vec{r}_{2}^{\prime}\right)=g_{2}^{T(s)}\left(\vec{r}_{1}, \vec{r}_{2} ; \vec{r}_{1}^{\prime}, \vec{r}_{2}^{\prime}\right)+\left[\left(\frac{\left(\vec{r}_{12^{\prime}} \vec{r}_{11^{\prime}}\right)}{\vec{r}_{11^{\prime}}^{2} \vec{r}_{12^{\prime}}^{2} \vec{r}_{1^{\prime} 2^{\prime}}^{2}}+\frac{1}{2 \vec{r}_{1^{\prime} 2^{\prime}}^{4}}\right.\right. \\
& \left.\left.+\frac{\vec{r}_{12}^{2}}{4 \vec{r}_{1^{\prime} 2^{\prime}}^{2}}\left(\frac{1}{\vec{r}_{11^{\prime}}^{2} \vec{r}_{22^{\prime}}^{2}}+\frac{1}{\vec{r}_{12^{\prime}}^{2} \vec{r}_{21^{\prime}}^{2}}\right)-\frac{\vec{r}_{12}^{2}}{4 \vec{r}_{11^{\prime}}^{2} \vec{r}_{22^{\prime}}^{2} \vec{r}_{12^{\prime}}^{2} \vec{r}_{21^{\prime}}^{2}}\right) \ln \left(\frac{\vec{r}_{11^{\prime}}^{2}}{\vec{r}_{12^{\prime}}^{2}}\right)+\left(1 \leftrightarrow 2,1^{\prime} \leftrightarrow 2^{\prime}\right)\right],
\end{aligned}
$$


where

$$
g_{2}^{T(s)}\left(\vec{r}_{1}, \vec{r}_{2} ; \vec{r}_{1}^{\prime}, \vec{r}_{2}^{\prime}\right)=g_{2}^{B C}\left(\vec{r}_{1}, \vec{r}_{2} ; \vec{r}_{1}^{\prime}, \vec{r}_{2}^{\prime}\right)
$$

is symmetric with respect to the replacement $\left(\vec{r}_{1}^{\prime} \leftrightarrow \vec{r}_{2}^{\prime}\right.$ ) (as well as with respect to the replacement $\left(\vec{r}_{1} \leftrightarrow \vec{r}_{2}\right)$ ), and the terms in the square brackets are antisymmetric, so that they can be omitted with account of the symmetry of impact factors. There is also another reason for omitting the first term: it does not depend on $\vec{r}_{2}$. In the following we will assume that this term is omitted. Note that using Eq. (16) we can rewrite $g_{0}^{T}=g_{0}$ in the form

$$
g_{0}^{T}\left(\vec{r}_{1}, \vec{r}_{2}, \vec{\rho}\right)=2 \pi \zeta(3) \delta(\vec{\rho})-g_{1}^{T}\left(\vec{r}_{1}, \vec{r}_{2}, \vec{\rho}\right) .
$$

Comparing Eqs. (37) and (40) with Eqs. (18) and (17) we see that up to the normalization points the functions $g_{1}^{T}$ and $g_{0}^{T}$ coincide with $g_{1}^{B C}$ and $g_{0}^{B C}$ correspondingly. Therefore, we conclude that the symmetrized gluon part of the Möbius form of the kernel can be written as

$$
\hat{K}-\left[\hat{K}^{B}, \hat{O}\right]=\hat{\mathcal{K}}+\frac{\alpha_{s}}{8 \pi} \beta_{0}\left[\hat{\mathcal{K}}^{B}, \ln \left(\hat{\vec{q}}_{1}^{2} \hat{\vec{q}}_{2}^{2}\right)\right]-\left[\hat{\mathcal{K}}^{B}, \hat{O}\right],
$$

where $\beta_{0}$ is the first coefficient of the beta-function, the kernel $\mathcal{K}$ is defined in Eq. (3) and the operator $\hat{O}$ in Eq. (25). It coincides (up to the difference in the renormalizations) with the gluon part of the kernel of the colour dipole approach found in Ref. [17] (with account of the correction given in Ref. [25]).

Since the Möbius form of the quark part of $\hat{K}$ coincides with the quark part of the linearized BK kernel [15, 16] calculated in the colour dipole model, and only gluons contribute to $\left[\hat{\mathcal{K}}^{B}, \hat{O}\right]$ (see Eq. (26)), it means that the the symmetrized Möbius form of the kernel (41) coincides with the kernel of the colour dipole model (up to the difference in the renormalization scales). Thus, the discrepancy between the BFKL and the colour dipole approaches is completely removed.

\section{Transformation to the quasi-conformal shape}

As can be seen from the representation (11) and from the explicit expressions for $g_{i}^{T}$, given by Eqs. (37)-(40), (201), the conformal invariance of the Möbius form of the kernel (41) is violated not only by the terms related to renormalization. However, from the results of Ref. [25] it is clear that we can transform the form (41) to the quasi-conformal kernel. Indeed, the transformation from the usual kernel for the evolution of colour dipoles to the kernel for the evolution of the "composite dipole operators" used in Ref. [25] has the same nature as the transformation (92). Let us show that the transformation $\hat{K} \rightarrow \hat{\mathcal{K}}^{Q C}=\hat{K}-\left[\hat{\mathcal{K}}^{B}, O_{1}\right]$, where

$\left\langle\vec{r}_{1} \vec{r}_{2}\left|\hat{O}_{1 M}\right| \vec{r}_{1}^{\prime} \vec{r}_{2}^{\prime}\right\rangle=\frac{\alpha_{s}(\mu) N_{c}}{4 \pi^{2}} \int d \vec{\rho} \frac{\vec{r}_{12}^{2}}{\vec{r}_{1 \rho}^{2} \vec{r}_{2 \rho}^{2}} \ln \left(\frac{\vec{r}_{12}^{2}}{\vec{r}_{1 \rho}^{2} \vec{r}_{2 \rho}^{2}}\right)\left[\delta\left(\vec{r}_{11^{\prime}}\right) \delta\left(\vec{r}_{2^{\prime} \rho}\right)+\delta\left(\vec{r}_{1^{\prime} \rho}\right) \delta\left(\vec{r}_{22^{\prime}}\right)-\delta\left(\vec{r}_{11^{\prime}}\right) \delta\left(r_{22^{\prime}}\right)\right]$, 
eliminates the nonconformal terms in $\hat{K}_{M}$ which are not proportional to the $\beta$-function.

Indeed, with the help of the integrals from Appendix A of Ref. [26], for the commutator of this operator with the Born part of the Möbius kernel (11) we obtain

$$
\begin{gathered}
\left\langle\vec{r}_{1} \vec{r}_{2}\left|\left[\hat{\mathcal{K}}_{M}^{B}, \hat{O}_{1 M}\right]\right| \vec{r}_{1}^{\prime} \vec{r}_{2}^{\prime}\right\rangle=-\frac{\alpha_{s}^{2}(\mu) N_{c}^{2}}{4 \pi^{4}}\left[\frac{\vec{r}_{12}^{2}}{\vec{r}_{11^{\prime}}^{2} \vec{r}_{22^{\prime}}^{2} \vec{r}_{1^{\prime} 2^{\prime}}^{2}} \ln \left(\frac{\vec{r}_{12}^{2} \vec{r}_{1^{\prime} 2^{\prime}}^{2}}{\vec{r}_{12^{\prime}}^{2} \vec{r}_{21^{\prime}}^{2}}\right)\right. \\
\left.+\pi \delta\left(\vec{r}_{11^{\prime}}\right) \frac{\vec{r}_{12}^{2}}{\vec{r}_{22^{\prime}}^{2} \vec{r}_{12^{\prime}}^{2}} \ln \left(\frac{\vec{r}_{12}^{2}}{\vec{r}_{22^{\prime}}^{2}}\right) \ln \left(\frac{\vec{r}_{12}^{2}}{\vec{r}_{12^{\prime}}^{2}}\right)+\pi \delta\left(r_{22^{\prime}}\right) \frac{\vec{r}_{12}^{2}}{\vec{r}_{11^{\prime}}^{2} \vec{r}_{21^{\prime}}^{2}} \ln \left(\frac{\vec{r}_{12}^{2}}{\vec{r}_{11^{\prime}}^{2}}\right) \ln \left(\frac{\vec{r}_{12}^{2}}{\vec{r}_{21^{\prime}}^{2}}\right)\right] .
\end{gathered}
$$

Then, using the functions $g_{i}^{T}$ (37)-(40) for the kernel $\hat{K}$, we obtain the functions $g_{i}^{Q C}$ for the kernel $\hat{\mathcal{K}}^{Q C}=\hat{K}-\left[\hat{\mathcal{K}}^{B}, O_{1}\right]$ :

$$
\begin{gathered}
g_{0}^{Q C}\left(\vec{r}_{1}, \vec{r}_{2} ; \vec{\rho}\right)=6 \pi \zeta(3) \delta(\vec{\rho})-g\left(\vec{r}_{1}, \overrightarrow{r_{2}} ; \vec{\rho}\right), \\
g_{1}^{Q C}\left(\vec{r}_{1}, \vec{r}_{2} ; \vec{r}_{2}^{\prime}\right)=\frac{11}{6} \frac{\vec{r}_{12}^{2}}{\vec{r}_{22^{\prime}}^{2} \vec{r}_{12^{\prime}}^{2}} \ln \left(\frac{\vec{r}_{12}^{2}}{r_{\mu}^{2}}\right)+\frac{11}{6}\left(\frac{1}{\vec{r}_{22^{\prime}}^{2}}-\frac{1}{\vec{r}_{12^{\prime}}^{2}}\right) \ln \left(\frac{\vec{r}_{22^{\prime}}^{2}}{\vec{r}_{12^{\prime}}^{2}}\right),
\end{gathered}
$$

where $\ln r_{\mu}^{2}$ is defined in Eq. (14), and

$$
\begin{gathered}
g_{2}^{Q C}\left(\vec{r}_{1}, \vec{r}_{2} ; \vec{r}_{1}^{\prime}, \vec{r}_{2}^{\prime}\right)=\frac{1}{\vec{r}_{1^{\prime} 2^{\prime}}^{4}}\left(\frac{\vec{r}_{11^{\prime}}^{2} \vec{r}_{22^{\prime}}^{2}-2 \vec{r}_{12}^{2} \vec{r}_{1^{\prime} 2^{\prime}}^{2}}{d} \ln \left(\frac{\vec{r}_{12^{\prime}}^{2} \vec{r}_{21^{\prime}}^{2}}{\vec{r}_{11^{\prime}}^{2} \vec{r}_{22^{\prime}}^{2}}\right)-1\right)+\frac{\vec{r}_{12}^{2}}{\vec{r}_{11^{\prime}}^{2} \vec{r}_{22^{\prime}}^{2} \vec{r}_{1^{\prime} 2^{\prime}}^{2}} \ln \left(\frac{\vec{r}_{12}^{2} \vec{r}_{1^{\prime} 2^{\prime}}^{2}}{\vec{r}_{12^{\prime}}^{2} \vec{r}_{21^{\prime}}^{2}}\right) \\
+\frac{1}{2 \vec{r}_{11^{\prime}}^{2} \vec{r}_{22^{\prime}}^{2}} \ln \left(\frac{\vec{r}_{12^{\prime}}^{2} \vec{r}_{21^{\prime}}^{2}}{\vec{r}_{11^{\prime}}^{2} \vec{r}_{22^{\prime}}^{2}}\right)\left(\frac{\vec{r}_{12}^{4}}{d}-\frac{\vec{r}_{12}^{2}}{\vec{r}_{1^{\prime} 2^{\prime}}^{2}}\right), \quad d=\vec{r}_{12^{\prime}}^{2} \vec{r}_{21^{\prime}}^{2}-\vec{r}_{11^{\prime}}^{2} \vec{r}_{22^{\prime}}^{2} .
\end{gathered}
$$

¿From the representation (11) and the expressions (44)-(46) it can be seen that the conformal invariance is violated only by the terms proportional to $11 / 6$. Remind that in the quark contribution the violation has the same form with $-n_{f} / 3\left(n_{f}\right.$ is the quark flavour number) instead of $11 / 6$, so that the total violation is proportional to the $\beta$-function. It means that the kernel $\hat{\mathcal{K}}^{Q C}=\hat{K}-\left[\hat{\mathcal{K}}^{B}, O_{1}\right]$ is quasi-conformal, i.e. nonconformal terms in its Möbius form have origin from the renormalization procedure.

The part of the gluon contribution to the Möbius form of $\hat{\mathcal{K}}^{Q C}$ symmetric with respect to the substitution $\left(\vec{r}_{1}^{\prime} \leftrightarrow \vec{r}_{2}^{\prime}\right)$ coincides with the corresponding contribution to the kernel for the evolution of the "composite dipole operators" obtained in Eq. (70) of Ref. [25], if one does not pay attention to the misprint in this equation (instead of $d^{2} z_{3} d^{2} z_{4} / z_{34}^{2}$ must be $d^{2} z_{3} d^{2} z_{4} / z_{34}^{4}$ ) and to the difference in the renormalization scales: $r_{\mu_{B C}}^{2}$ instead of our $r_{\mu}^{2}(14)$, being

$$
r_{\mu_{B C}}^{2}=\frac{r_{\mu}^{2}}{4 e^{2 \psi(1)}}, \quad \mu_{B C}^{2}=\frac{\mu^{2}}{4 e^{2 \psi(1)}} .
$$

As was pointed out in Ref. [26], we think that this difference arose because the renormalization scheme used in Refs. [17] and [25] is not equivalent to the conventional $\overline{M S}$ renormalization scheme defined in the momentum space. 


\section{Möbius forms for total (quasi-)conformal kernels}

In this section we present the Möbius form for the total quasi-conformal BFKL kernels in QCD and extended supersymmetric Yang-Mills theories with arbitrary $N$. In all these theories the quasi-conformal kernel $\hat{\mathcal{K}}^{Q C}$ is defined by the relation

$$
\hat{\mathcal{K}}^{Q C}=\hat{\mathcal{K}}+\frac{\alpha_{s}}{8 \pi} \beta_{0}\left[\hat{\mathcal{K}}^{B}, \ln \left(\hat{\vec{q}}_{1}^{2} \hat{\vec{q}}_{2}^{2}\right)\right]-\left[\hat{\mathcal{K}}^{B}, \hat{O}+\hat{O}_{1}\right]
$$

where $\beta_{0}$ is the first coefficient of the beta-function for the corresponding theory, the operators $\hat{O}$ and $\hat{O}_{1}$ are defined in Eqs. (25) and (42) respectively. For QCD the kernel $\mathcal{K}$ is the usual BFKL kernel defined in the momentum representation (see Eq. (3ib)). For SUSY Yang-Mills theories in the $\overline{M S}$ renormalization scheme it is obtained [22] from the QCD kernel by the change of the coefficients $n_{f}$ with $n_{M} N_{c}\left(n_{M}\right.$ is the number of gluinos, $\left.n_{M}=N\right)$ in the "non-Abelian" part and $n_{f}$ with $-n_{M} N_{c}^{3}$ in the "Abelian" part of the quark contribution, and by addition of the contribution of $n_{s}$ scalars $\left(n_{S}=2(N-1)\right)$. The latter contribution is defined in the momentum space by Eqs. (19)-(22) and (28) in Ref. [23].

In the QCD case, using the gluon contribution from the previous section and taking the quark part from Refs. [10, 18], for the Möbius form of the quasi-conformal kernel (48) we get

$$
\begin{gathered}
g_{0}^{Q C(Q C D)}\left(\vec{r}_{1}, \vec{r}_{2} ; \vec{\rho}\right)=6 \pi \zeta(3) \delta(\vec{\rho})-g_{1}^{Q C(Q C D)}\left(\vec{r}_{1}, \vec{r}_{2} ; \vec{\rho}\right), \\
g_{1}^{Q C(Q C D)}\left(\vec{r}_{1}, \vec{r}_{2} ; \vec{r}_{2}^{\prime}\right) \\
=\frac{\vec{r}_{12}^{2}}{\vec{r}_{22^{\prime}}^{2} \vec{r}_{12^{\prime}}^{2}}\left[\frac{67}{18}-\zeta(2)-\frac{5 n_{f}}{9 N_{c}}+\frac{\beta_{0}}{2 N_{c}} \ln \left(\frac{\vec{r}_{12}^{2} \mu^{2}}{4 e^{2 \psi(1)}}\right)+\frac{\beta_{0}}{2 N_{c}} \frac{\vec{r}_{12^{\prime}}^{2}-\vec{r}_{22^{\prime}}^{2}}{\vec{r}_{12}^{2}} \ln \left(\frac{\vec{r}_{22^{\prime}}^{2}}{\vec{r}_{12^{\prime}}^{2}}\right)\right], \\
g_{2}^{Q C(Q C D)}\left(\vec{r}_{1}, \vec{r}_{2}^{2} ; \vec{r}_{1}^{\prime}, \vec{r}_{2}^{\prime}\right)=\frac{1}{\vec{r}_{1^{\prime} 2^{\prime}}^{4}}\left(\frac{\vec{r}_{11^{\prime}}^{2} \vec{r}_{22^{\prime}}^{2}-2 \vec{r}_{12}^{2} \vec{r}_{1^{\prime} 2^{\prime}}^{2}}{d} \ln \left(\frac{\vec{r}_{12^{\prime}}^{2} \vec{r}_{21^{\prime}}^{2}}{\vec{r}_{11^{\prime}}^{2} \vec{r}_{22^{\prime}}^{2}}\right)-1\right)\left(1+\frac{n_{f}}{N_{c}^{3}}\right) \\
+\left(\frac{3 n_{f}}{2 N_{c}^{3}} \frac{\vec{r}_{12}^{2}}{\vec{r}_{1^{\prime} 2^{\prime}}^{2} d}+\frac{1}{2 \vec{r}_{11^{\prime}}^{2} \vec{r}_{22^{\prime}}^{2}}\left(\frac{\vec{r}_{12}^{4}}{d}-\frac{\vec{r}_{12}^{2}}{\vec{r}_{1^{\prime} 2^{\prime}}^{2}}\right)\right) \ln \left(\frac{\vec{r}_{12^{\prime}}^{2} \vec{r}_{21^{\prime}}^{2}}{\vec{r}_{11^{\prime}}^{2} \vec{r}_{22^{\prime}}^{2}}\right)+\frac{\vec{r}_{12}^{2}}{\vec{r}_{11^{\prime}}^{2} \vec{r}_{22^{\prime}}^{2} \vec{r}_{1^{\prime} 2^{\prime}}^{2}} \ln \left(\frac{\vec{r}_{12}^{2} \vec{r}_{1^{\prime} 2^{\prime}}^{2}}{\vec{r}_{12^{\prime}}^{2} \vec{r}_{21^{\prime}}^{2}}\right) .
\end{gathered}
$$

Here $\beta_{0}=\frac{11}{3} N_{c}-\frac{2}{3} n_{f}$. With account of $r_{1} \leftrightarrow r_{2}$ symmetrization this result coincides with the result of Ref. 25] up to the different $\mu$ (47).

Since the dimensional regularization violates the supersymmetry, the regularization which is commonly used in supersymmetric theories is the dimensional reduction. So, we take the scalar and fermion contributions from Ref. [23], add the gluon one found in the previous section, and express our result in the dimensional reduction scheme, which differs from the $\overline{M S}$ scheme by the finite charge renormalization (see Ref. [23] for details):

$$
\alpha_{s} \rightarrow \alpha_{s}\left(1-\frac{\alpha_{s} N_{c}}{12 \pi}\right)
$$


Thus we find

$$
\begin{gathered}
g_{S U S Y}^{0}\left(\vec{r}_{1}, \vec{r}_{2} ; \vec{\rho}\right)=6 \pi \zeta(3) \delta(\vec{\rho})-g_{S U S Y}\left(\vec{r}_{1}, \vec{r}_{2} ; \vec{\rho}\right) \\
g_{S U S Y}\left(\vec{r}_{1}, \vec{r}_{2} ; \vec{r}_{2}^{\prime}\right) \\
=\frac{\vec{r}_{12}^{2}}{\vec{r}_{22^{\prime}}^{2} \vec{r}_{12^{\prime}}^{2}}\left[\frac{32}{9}-\zeta(2)-\frac{5 n_{M}+2 n_{S}}{9}+\frac{\beta_{0}}{2 N_{c}} \ln \left(\frac{\vec{r}_{12}^{2} \mu^{2}}{4 e^{2 \psi(1)}}\right)+\frac{\beta_{0}}{2 N_{c}} \frac{\vec{r}_{12^{\prime}}^{2}-\vec{r}_{22^{\prime}}^{2}}{\vec{r}_{12}^{2}} \ln \left(\frac{\vec{r}_{22^{\prime}}^{2}}{\vec{r}_{12^{\prime}}^{2}}\right)\right] \\
g_{S U S Y}\left(\vec{r}_{1}, \vec{r}_{2} ; \vec{r}_{1}^{\prime}, \vec{r}_{2}^{\prime}\right)=\frac{1}{\vec{r}_{1^{\prime} 2^{\prime}}^{4}}\left(\frac{\vec{r}_{11^{\prime}}^{2} \vec{r}_{22^{\prime}}^{2}-2 \vec{r}_{12}^{2} \vec{r}_{1^{\prime} 2^{\prime}}^{2}}{d} \ln \left(\frac{\vec{r}_{12^{\prime}}^{2} \vec{r}_{21^{\prime}}^{2}}{\vec{r}_{11^{\prime}}^{2} \vec{r}_{22^{\prime}}^{2}}\right)-1\right)\left(1-n_{M}+\frac{n_{S}}{2}\right) \\
+\left(\frac{\left(2 n_{S}-3 n_{M}\right)}{2 \vec{r}_{1^{\prime} 2^{\prime}}^{2}} \frac{\vec{r}_{12}^{2}}{d}+\frac{1}{2 \vec{r}_{11^{\prime}}^{2} \vec{r}_{22^{\prime}}^{2}}\left(\frac{\vec{r}_{12}^{4}}{d}-\frac{\vec{r}_{12}^{2}}{\vec{r}_{1^{\prime} 2^{\prime}}^{2}}\right)\right) \ln \left(\frac{\vec{r}_{12^{\prime}}^{2} \vec{r}_{21^{\prime}}^{2}}{\vec{r}_{11^{\prime}}^{2} \vec{r}_{22^{\prime}}^{2}}\right)+\frac{\vec{r}_{12}^{2}}{\vec{r}_{11^{\prime}}^{2} \vec{r}_{22^{\prime}}^{2} \vec{r}_{1^{\prime} 2^{\prime}}^{2}} \ln \left(\frac{\vec{r}_{12}^{2} \vec{r}_{1^{\prime} 2^{\prime}}^{2}}{\vec{r}_{12^{\prime}}^{2} \vec{r}_{21^{\prime}}^{2}}\right), \\
d=\vec{r}_{12^{\prime}}^{2} \vec{r}_{21^{\prime}}^{2}-\vec{r}_{11^{\prime}}^{2} \vec{r}_{22^{\prime}}^{2}, \quad \beta_{0}=\left(\frac{11}{3}-\frac{2 n_{M}}{3}-\frac{n_{S}}{6}\right) N_{c} .
\end{gathered}
$$

Finally for $N=4$ SUSY theory, we put $n_{S}=6, n_{M}=4, \beta_{0}=0$ and write

$$
\begin{gathered}
\left\langle\vec{r}_{1} \vec{r}_{2}\left|\hat{\mathcal{K}}_{M}^{Q C}\right| \vec{r}_{1}^{\prime} \vec{r}_{2}^{\prime}\right\rangle_{N=4} \\
=\frac{\alpha_{s} N_{c}}{2 \pi^{2}} \int d \vec{\rho}_{\frac{r_{12}}{\vec{r}_{1 \rho}^{2} \vec{r}_{2 \rho}^{2}}}^{2}\left[\delta\left(\vec{r}_{11^{\prime}}\right) \delta\left(\vec{r}_{2^{\prime} \rho}\right)+\delta\left(\vec{r}_{1^{\prime} \rho}\right) \delta\left(\vec{r}_{22^{\prime}}\right)-\delta\left(\vec{r}_{11^{\prime}}\right) \delta\left(r_{22^{\prime}}\right)\right]\left(1-\frac{\alpha_{s} N_{c} \zeta(2)}{2 \pi}\right) \\
+\frac{\alpha_{s}^{2} N_{c}^{2}}{4 \pi^{4}}\left[\frac{\ln \left(\frac{\vec{r}_{12^{\prime}}^{2} \vec{r}_{21^{\prime}}^{2}}{\vec{r}_{11^{\prime}}^{2} \vec{r}_{22^{\prime}}^{2}}\right)}{2 \vec{r}_{11^{\prime}}^{2} \vec{r}_{22^{\prime}}^{2}}\left(\frac{\vec{r}_{12}^{4}}{\vec{r}_{12^{\prime}}^{2} \vec{r}_{21^{\prime}}^{2}-\vec{r}_{11^{\prime}}^{2} \vec{r}_{22^{\prime}}^{2}}-\frac{\vec{r}_{12}^{2}}{\vec{r}_{12^{\prime}}^{2}}\right)+\frac{\vec{r}_{12}^{2} \ln \left(\frac{\vec{r}_{12}^{2} \vec{r}_{1^{\prime} 2^{\prime}}^{2}}{\vec{r}_{12^{\prime}}^{2} \vec{r}_{1^{\prime}}^{2}}\right)}{\vec{r}_{11^{\prime}}^{2} \vec{r}_{22^{\prime}}^{2} \vec{r}_{1^{\prime} 2^{\prime}}^{2}}+6 \pi^{2} \zeta(3) \delta\left(\vec{r}_{11^{\prime}}\right) \delta\left(r_{22^{\prime}}\right)\right] .
\end{gathered}
$$

This kernel is conformally invariant and coincides with the linearized BK kernel obtained in Ref. 25] with account of $r_{1} \leftrightarrow r_{2}$ symmetrization.

\section{Conclusion}

The main results of this paper are the following. First, we demonstrated that the discrepancy between the gluon contribution to the Möbius form of the BFKL kernel, calculated in Ref. [21], and the corresponding contribution to the kernel of the colour dipole model, calculated in Refs. [17] and [25], can be removed due to the ambiguity of the kernels in the next-to-leading order, which allows the transformations (9). It was explicitly shown that the symmetrized gluon part of the Möbius form of the kernel (41) coincides (up to the difference in the renormalization scales (47)) with the gluon part of the kernel of the colour dipole approach found in Ref. [17] (with account of the correction of Ref. [25]). In our opinion, the scales differ because the 
renormalization scheme used in Refs. [17] and [25] is not equivalent to the conventional $\overline{M S}$ renormalization scheme defined in the momentum space.

Second, using the ambiguity mentioned above and the results of Ref. [25], we constructed the quasi-conformal kernel (48) and found the Möbius form of this kernel in QCD and $N$-extended supersymmetric Yang-Mills theories. The nonconformal terms in this form are proportional to the first coefficient of the $\beta$-function. At $N=4$ the Möbius form is conformally invariant and coincides with the result of Ref. [25].

\section{Acknowledgment}

V.S.F. thanks the Dipartimento di Fisica dell'Università della Calabria and the Istituto Nazionale di Fisica Nucleare, Gruppo Collegato di Cosenza, for the warm hospitality while part of this work was done and for the financial support.

\section{References}

[1] V. S. Fadin, E. A. Kuraev, and L. N. Lipatov, Phys. Lett. B60 (1975) 50. E. A. Kuraev, L. N. Lipatov, and V. S. Fadin, Sov. Phys. JETP 44 (1976) 443 [Zh. Eksp. Teor. Fiz. 71 (1976) 840]. E. A. Kuraev, L. N. Lipatov and, V. S. Fadin, Sov. Phys. JETP 45 (1977) 199 [Zh. Eksp. Teor. Fiz. 72 (1977) 377]. I. I. Balitsky and L. N. Lipatov, Sov. J. Nucl. Phys. 28 (1978) 822 [Yad. Fiz. 28 (1978) 1597].

[2] N.N. Nikolaev and B.G. Zakharov, Z. Phys. C64 (1994) 631;

N.N. Nikolaev, B.G. Zakharov, and V.R. Zoller, JETP Lett. 59 (1994) 6;

A.H. Mueller, Nucl. Phys.B415 (1994) 373;

A.H. Mueller and B. Patel, Nucl. Phys. B425 (1994) 471.

[3] V. S. Fadin and L. N. Lipatov, Phys. Lett. B429 (1998) 127 arXiv:hep-ph/9802290].

[4] M. Ciafaloni and G. Camici, Phys. Lett. B430 (1998) 349.

[5] V.S. Fadin, R. Fiore, and A. Papa, Phys. Rev. D60 (1999) 074025.

[6] V. S. Fadin and D. A. Gorbachev, JETP Lett. 71 (2000) 222 [Pisma Zh. Eksp. Teor. Fiz. 71 (2000) 322]; Phys. Atom. Nucl. 63 (2000) 2157 [Yad. Fiz. 63 (2000) 2253].

[7] V. S. Fadin and R. Fiore, Phys. Lett. B610 (2005) 61 [Erratum-ibid. B621 (2005) 61] arXiv:hep-ph/0412386]; Phys. Rev. D72 (2005) 014018 [arXiv:hep-ph/0502045]. 
[8] L.N. Lipatov, Sov. Phys. JETP 63 (1986) 904 [Zh. Eksp. Teor. Fiz. 90 (1986) 1536].

[9] J. Bartels, L. N. Lipatov, and G. P. Vacca, Nucl. Phys. B706 (2005) 391 arXiv:hep-ph/0404110.

[10] V. S. Fadin, R. Fiore, and A. Papa, Nucl. Phys. B769(2007) 108 arXiv:hep-ph/0612284.

[11] L. V. Gribov, E. M. Levin, and M. G. Ryskin, Phys. Rep. 176 (1983) 1.

[12] I. Balitsky, Nucl. Phys. B463 (1996) 99;

[13] Yu. Kovchegov, Phys. Rev. D60 (1999) 034008.

[14] L. McLerran and R. Venugopalan, Phys. Rev. D49 (1994) 2233; ibid. D49 (1994) 3352; ibid D50 (1994) 2225;

E. Iancu, A. Leonidov and L. McLerran, Phys. Lett. B510 (2001) 133; Nucl. Phys. A692 (2001) 583;

E. Ferreiro, E. Iancu, A. Leonidov, and L. McLerran, Nucl. Phys. A701 (2002) 489;

J. Jalilian-Marian, A. Kovner, A. Leonidov and H. Weigert, Nucl. Phys. B504 (1997) 415; Phys. Rev. D59 (1999) 014014;

[15] Y. V. Kovchegov and H. Weigert, Nucl. Phys. A784 (2007) 188.

[16] I. Balitsky, Phys. Rev. D75 (2007) 014001 arXiv:hep-ph/0609105.

[17] I. Balitsky and G. A. Chirilli, Phys. Rev. D77 (2008)014019 [arXiv:0710.4330 [hep-ph]].

[18] V. S. Fadin, R. Fiore, and A. Papa, Phys. Lett. B647 (2007) 179 arXiv:hep-ph/0701075].

[19] V. N. Gribov, L. N. Lipatov, and G. V. Frolov, Sov. J. Nucl. Phys. 12 (1971) 543 [Yad. Fiz. 12 (1970) 994].

[20] H. Cheng and T. T. Wu, Phys. Rev. D1 (1970) 2775.

[21] V. S. Fadin, R. Fiore, A. V. Grabovsky, and A. Papa, Nucl. Phys. B784, 49 (2007) arXiv:0705.1885 [hep-ph]].

[22] A. V. Kotikov and L. N. Lipatov, Nucl. Phys. B582 (2000) 19 [arXiv:hep-ph/0004008].

[23] V. S. Fadin and R. Fiore, Phys. Lett. B661 (2008) 139 [arXiv:0712.3901 [hep-ph]].

[24] L. Cornalba, M. S. Costa and J. Penedones, arXiv:0911.0043 [hep-th]; JHEP 0806, 048 (2008), arXiv:0801.3002 [hep-th]]. 
[25] I. Balitsky and G. A. Chirilli, Nucl. Phys. B822 (2009) 45 [arXiv:0903.5326 [hep-ph]].

[26] V. S. Fadin, R. Fiore and A. V. Grabovsky, Nucl. Phys. B820 (2009) 334 arXiv:0904.0702 [hep-ph]].

[27] V.S. Fadin and R. Fiore, Phys. Lett. B440 (1998) 359. 